\title{
Precision measurement of the form factors of the charged kaon semileptonic decays $K^{ \pm} \rightarrow \pi_{l 3}^{ \pm}$
}

\author{
Mauro Piccini*广 \\ INFN - Sezione di Perugia \\ E-mail: Mauro.Piccini@pg.infn.it
}

\begin{abstract}
The NA48/2 experiment has presented a final result of the charged kaon semileptonic decays form factors measurement based on 4.28 million $K^{ \pm} \rightarrow \pi^{0} e^{ \pm} v\left(\mathrm{~K}_{e 3}\right)$ and 2.91 million $K^{ \pm} \rightarrow \pi^{0} \mu^{ \pm} v$ $\left(\mathrm{K}_{\mu 3}\right)$ selected decays, collected in 2004. The result is competitive with other measurements in $\left(\mathrm{K}_{\mu 3}\right)$ mode and has a smallest uncertainty for $\left(\mathrm{K}_{e 3}\right)$, that leads to the most precise combined $\left(\mathrm{K}_{l 3}\right)$ result and allows to reduce the form factor uncertainty on the extraction of $\left|\mathrm{V}_{U S}\right|$.
\end{abstract}

The European Physical Society Conference on High Energy Physics

5-12 July, 2017

Venice

* Speaker.

${ }^{\dagger}$ On behalf of the NA48/2 Collaboration: G. Anzivino, R. Arcidiacono, W. Baldini, S. Balev, J.R. Batley, M. Behler, S. Bifani, C. Biino, A. Bizzeti, B. Bloch-Devaux, G. Bocquet, N. Cabibbo, M. Calvetti, N. Cartiglia, A. Ceccucci, P. Cenci, C. Cerri, C. Cheshkov, J.B. Ch'eze, M. Clemencic, G. Collazuol, F. Costantini, A. Cotta Ramusino, D. Coward, D. Cundy, A. Dabrowski, P. Dalpiaz, C. Damiani, M. De Beer, J. Derr İĄe, H. Dibon, L. DiLella, N. Doble, K. Eppard, V. Falaleev, R. Fantechi, M. Fidecaro, L. Fiorini, M. Fiorini, T. Fonseca Martin, P.L. Frabetti, L. Gatignon, E. Gersabeck, A. Gianoli, S. Giudici, A. Gonidec, E. Goudzovski, S. Goy Lopez, M. Holder, P. Hristov, E. Iacopini, E. Imbergamo, M. Jeitler, G. Kalmus, V. Kekelidze, K. Kleinknecht, V. Kozhuharov, W. Kubischta, G. Lamanna, C. Lazzeroni, M. Lenti, L. Litov, D. Madigozhin, A. Maier, I. Mannelli, F. Marchetto, G. Marel, M. Markytan, P. Marouelli, M. Martini, L. Masetti, E. Mazzucato, A. Michetti, I. Mikulec, N. Molokanova, E. Monnier, U. Moosbrugger, C. Morales Morales, D.J. Munday, A. Nappi, G. Neuhofer, A. Norton, M. Patel, M. Pepe, A. Peters, F. Petrucci, M.C. Petrucci, B. Peyaud, M. Piccini, G. Pierazzini, I. Polenkevich, Yu. Potrebenikov, M. Raggi, B. Renk, P. Rubin, G. Ruggiero, M. Savrié, M. Scarpa, M. Shieh, M.W. Slater, M. Sozzi, S. Stoynev, E. Swallow, M. Szleper, M. Valdata-Nappi, B. Vallage, M. Velasco, M. Veltri, S. Venditti, M. Wache, H. Wahl, A. Walker, R. Wanke, L. Widhalm, A. Winhart, R. Winston, M.D. Wood, S.A. Wotton, A. Zinchenko, M. Ziolkowski. 


\section{Introduction}

The main purpose of the NA48/2 experiment at the CERN SPS was the search for direct CP violation in charge Kaons decay to three pions[1]; the experiment was collecting data in 2003 and 2004. A detailed description of the NA48 detector is available elsewhere [2]. The main difference of the NA48/2 setup was the replacement of the neutral kaon beams with two high intensity secondary beams $\left(K^{+}\right.$and $\left.K^{-}\right)$with a momentum of $60 \mathrm{GeV} / \mathrm{c}$ and a $\frac{\delta p}{p_{K}}=3.8 \%$ (rms). The two beams were simultaneous, generated from the same primary proton beam (400 Gev/c momentum) extracted from the SPS. The ration between the $K^{+}$and $K^{-}$fluxes was 1.8 and the rate of kaon decays was about $100 \mathrm{KHz}$. The data used for the form factor (FF) analysis were collected in 2004 during a dedicated run with a minimum bias trigger setup which only required at least one charged track crossing the charged hodoscope (CHOD) and an energy deposit of at least $10 \mathrm{GeV}$ in the Liquid Krypton Electromagnetic Calorimeter (LKr). Nearly $480 \times 10^{6}$ triggered events have been recorded during such special run.

\section{Semileptonic Kaon decays}

The $\mathrm{K}_{l 3}$ decay width in the absence of radiative corrections can be represented by the Dalitz plot density depending on $\mathrm{E}_{l}$ and $\mathrm{E}_{\pi}$, the lepton and pion energies in the kaon rest frame [3]:

$$
\frac{d^{2} \Gamma}{d E_{l} d E_{\pi}}=N\left[A f_{+}^{2}(t)+B f_{+}(t) f_{-}(t)+C f_{-}^{2}(t)\right]
$$

where $t=M_{l v}^{2}=\left(P_{K}-P_{\pi}\right)^{2}=m_{K}^{2}+m_{\pi}^{2}-2 m_{K} E_{\pi}, \mathrm{N}$ is a normalization constant and $f_{-}(t)=$ $\left(f_{+}(t)-f_{0}(t)\right)\left(m_{K}^{2}-m_{\pi}^{2}\right) / t . f_{+}(t)$ and $f_{0}(t)$ are the vector and scalar $\mathrm{K}_{l 3}$ form factors, respectively, $m_{K}$ is the mass of the charged kaon, $m_{\pi}$ is the mass of the neutral pion. $A, B$ and $C$ are kinematic factors:

$$
\begin{aligned}
& A=m_{K}\left[2 E_{l} E_{v}-m_{K}\left(E_{\pi}^{M A X}-E_{\pi}\right)\right]+m_{l}^{2}\left[\left(E_{\pi}^{M A X}-E_{\pi}\right) / 4-E_{v}\right] \\
& B=m_{l}^{2}\left[E_{v}-\left(E_{\pi}^{M A X}-E_{\pi}\right) / 2\right] \\
& C=m_{l}^{2}\left(E_{\pi}^{M A X}-E_{\pi}\right) / 4,
\end{aligned}
$$

where $E_{\pi}^{M A X}=\left(m_{K}^{2}+m_{\pi}^{2}-m_{l}^{2}\right) /\left(2 m_{K}\right)\left(m_{l}\right.$ is the lepton mass), and $E_{\mu}=m_{K}-E_{l}-E_{\pi}$ is the neutrino energy in the kaon rest frame. Giving the $m_{l}^{2}$ factor the terms $B$ and $C$ are negligible in the $K_{e 3}$ decay, for this channel only the vector form factor participates in the Dalitz plot description.

In Table 1 the definitions of the form factor parametrizations used in the analysis are shown: the quadratic parametrization [4] (fit parameters $\lambda_{+}^{\prime}, \lambda_{+}^{\prime \prime}, \lambda_{0}^{\prime}$ ), the pole parametrization [5] (fit parameters $\left.M_{V}, M_{S}\right)$ and the dispersive parametrization [6] (fit parameters $\left(\Lambda_{+}, \ln [C]\right.$ ).

\section{Selection and reconstruction of $\mathbf{K}_{e 3}$ and $\mathbf{K}_{\mu 3}$ samples}

The longitudinal position of the decay vertex of the charged kaon can be computed in two different ways: the first consists on the computation of the closest distance of approach between the nominal beam direction and the lepton track (reconstructed by the spectrometer), the second 


\begin{tabular}{|l|c|c|}
\hline Type of parametrization & $f_{+}(t)$ & $f_{0}(t)$ \\
\hline Quadratic & $1+\lambda_{+}^{\prime} t / m_{\pi}^{2}+\frac{1}{2} \lambda_{+}^{\prime \prime}\left(t / m_{\pi}^{2}\right)^{2}$ & $1+\lambda_{0}^{\prime} t / m_{\pi}^{2}$ \\
\hline Pole & $\frac{M_{V}^{2}}{M_{V}^{2}-t}$ & $\frac{M_{S}^{2}}{M_{S}^{2}-t}$ \\
\hline Dispersive & $e^{\frac{\left(\Lambda_{+}+H(t)\right) t}{m_{\pi}^{2}}}$ & $e^{\frac{(l n \mid C)-G(t) t}{m_{K}^{2}-m_{\pi}^{2}}}$ \\
\hline
\end{tabular}

Table 1: Parametrizations used in the present analysis to fit the form factor slopes.

exploits the longitudinal position of the $\pi^{0}$ decay, reconstructed only from $\mathrm{LKr}$ data assuming PDG[4] value for the $p i^{0}$ mass. In the present analysis the latter option is used in order to avoid a known transverse bias present in the first method. Once the $z$ is fixed, the $x$ and $y$ positions of the kaon decay vertex is taken from the nominal beam direction reconstructed from $K^{ \pm} \rightarrow \pi^{ \pm} \pi^{+} \pi^{-}$ decays. The full reconstruction of the event, taking into account the position of the decay vertex and the $\pi^{0}$ and lepton momenta, produces two solutions for the kaon momentum, the one with the closest value to the nominal beam momentum is chosen. The difference between the reconstructed kaon momentum and the nominal one is request to be less than $7.5 \mathrm{GeV} / \mathrm{c}$, otherwise the event is discarded.

To select a good $\pi^{0} \rightarrow \gamma \gamma$ candidate two clusters are request in the LKr with energy greater than $3 \mathrm{GeV}$, the distance between the two cluster has to be greater than $20 \mathrm{~cm}$ and the distance from the closest impact point of a track in the LKr has to be greater than $15 \mathrm{~cm}$. The total energy of the $\pi^{0}$ has to be greater than $15 \mathrm{GeV}$ to ensure a good efficiency of the trigger for the selected events. To reduce background from $K^{ \pm} \rightarrow \pi^{ \pm} \pi^{0} \pi^{0}$ no extra-photons are has to be present in time ( $\pm 5 \mathrm{~ns}$ ).

The preliminary requests on lepton track are: the track has to be in time with the $\pi^{0}( \pm 10$ $\mathrm{ns})$, and distance of the track from the nominal beam axis greater than $15 \mathrm{~cm}$ at each drift chamber plane. No extra-tracks in time ( $\pm 8 \mathrm{~ns})$ must be present. Then different cuts are applied to $e^{ \pm}$and $\mu^{ \pm}$.

For $K_{e 3}$ selection the track momentum has requested to be greater than $5 \mathrm{GeV} / \mathrm{c}$ and the ratio $E / p$ of the energy $E$ measured in the LKr and momentum $p$ measured from the spectrometer has to be greater than 0.9. A requirement on the reconstructed neutrino transverse momentum is also applied: $P_{T}^{n u}>0.03 \mathrm{GeV} / \mathrm{c}$.

For $K_{\mu 3}$ selection the track momentum has requested to be greater than $10 \mathrm{GeV} / \mathrm{c}$ (to ensure a good efficiency of the muon detector), the ratio $E / p$ is required to be lower than 0.9 and a muon hit associated with the track must be present in the muon detector. Selective cuts are then applied to suppress the background from the decays $K^{ \pm} \rightarrow \pi^{ \pm} \pi^{0}$ and $K^{ \pm} \rightarrow \pi^{ \pm} \pi^{0} \pi^{0}$ with a subsequent $p i^{ \pm} \rightarrow \mu^{ \pm} v$ decay.

The Dalitz plots of the selected events are shown in Fig. 1 with a binning of $5 \times 5 \mathrm{MeV}$. They are used for all the further fits after correction for residual background. The residual background are estimated using the Monte-Carlo and their contributions are subtracted in the Dalitz distributions. The total number of events on the final samples are 4.278 million of $\mathrm{K}_{e 3}$ and 2.907 million of $\mathrm{K}_{\mu 3}$. 

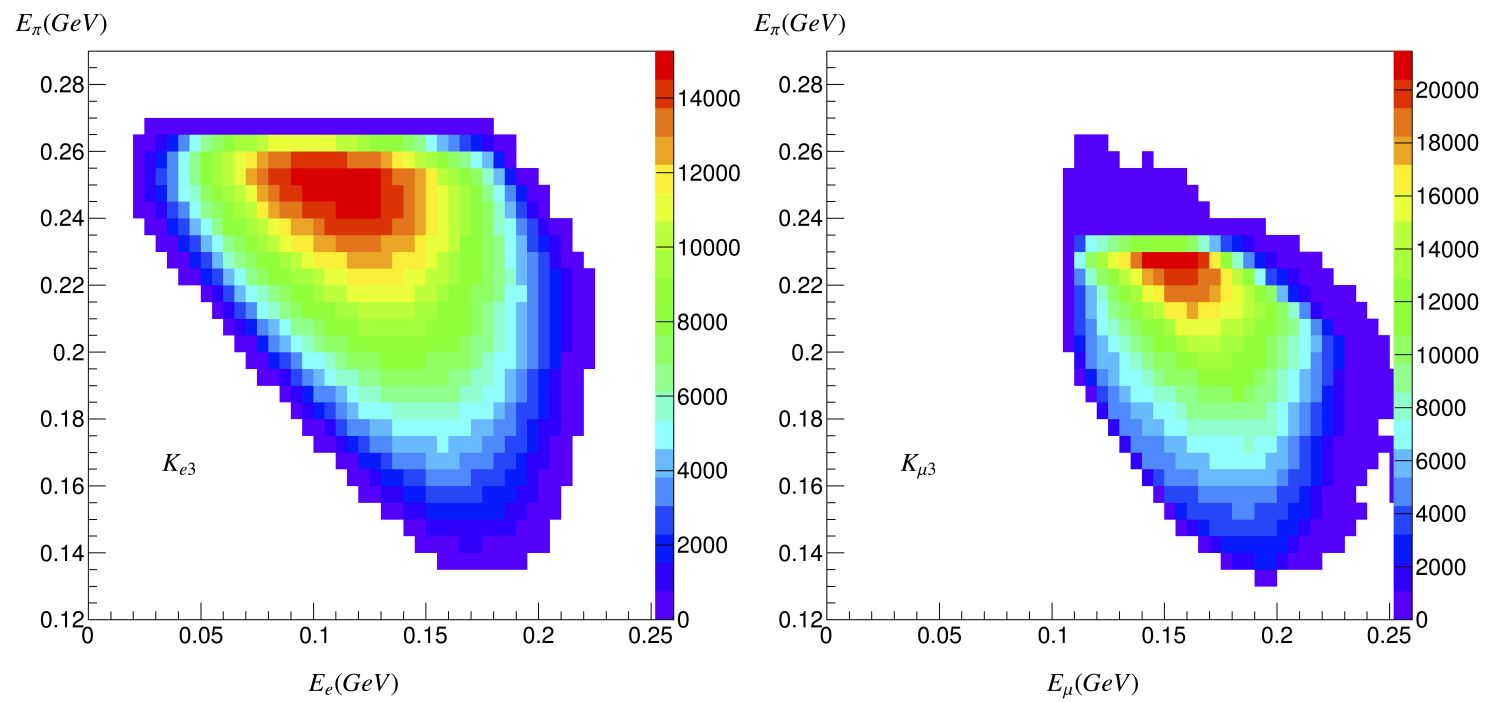

Figure 1: Reconstructed Dalitz plots for $\mathrm{K}_{e 3}$ (left) and $\mathrm{K}_{\mu 3}$ (right).

\section{Results and conclusions}

The measurements of the form factors are obtained with the minimization of a $\chi^{2}$ given by the bin by bin difference between the experimental (background-corrected) Dalitz plot and the Monte-Carlo one. The semileptonic radiative Monte Carlo samples have been simulated with the KLOE generator [7]. Fit results and contributions to systematic uncertainty for quadratic, pole and dispersive parametrization are shown in Table 2.

\begin{tabular}{|l|c|c|c|}
\hline Quadratic & $\lambda_{+}^{\prime}\left(\times 10^{3}\right)$ & $\lambda_{+}^{\prime \prime}\left(\times 10^{3}\right)$ & $\lambda_{0}\left(\times 10^{3}\right)$ \\
\hline$K_{\mu 3}$ & $23.32 \pm 3.08_{\text {stat }} \pm 3.50_{\text {syst }}$ & $2.14 \pm 1.06_{\text {stat }} \pm 0.96_{\text {syst }}$ & $14.33 \pm 1.11_{\text {stat }} \pm 1.25_{\text {syst }}$ \\
$K_{e 3}$ & $23.52 \pm 0.78_{\text {stat }} \pm 1.29_{\text {syst }}$ & $1.60 \pm 0.30_{\text {stat }} \pm 0.39_{\text {syst }}$ & \\
$K_{l 3}$ & $23.35 \pm 0.75_{\text {stat }} \pm 1.23_{\text {syst }}$ & $1.73 \pm 0.29_{\text {stat }} \pm 0.41_{\text {syst }}$ & $14.90 \pm 0.55_{\text {stat }} \pm 0.80_{\text {syst }}$ \\
\hline \hline Pole & $M_{V}\left(\mathrm{MeV} / \mathrm{c}^{2}\right)$ & $M_{S}\left(\mathrm{MeV} / \mathrm{c}^{2}\right)$ & \\
\hline$K_{\mu 3}$ & $879.1 \pm 8.1_{\text {stat }} \pm 13.5_{\text {syst }}$ & $1196.4 \pm 18.1_{\text {stat }} \pm 28.8_{\text {syst }}$ & \\
$K_{e 3}$ & $896.8 \pm 3.4_{\text {stat }} \pm 7.6_{\text {syst }}$ & & \\
$K_{l 3}$ & $894.3 \pm 3.2_{\text {stat }} \pm 5.4_{\text {syst }}$ & $1185.5 \pm 16.6_{\text {stat }} \pm 35.5_{\text {syst }}$ & \\
\hline \hline Dispersive & $\Lambda_{+}\left(\times 10^{3}\right)$ & $\ln [\mathrm{C}]\left(\times 10^{3}\right)$ & \\
\hline$K_{\mu 3}$ & $23.55 \pm 0.50_{\text {stat }} \pm 0.97_{\text {syst }}$ & $186.68 \pm 5.12_{\text {stat }} \pm 9.23_{\text {syst }}$ & \\
$K_{e 3}$ & $22.54 \pm 0.20_{\text {stat }} \pm 0.62_{\text {syst }}$ & & \\
$K_{l 3}$ & $22.67 \pm 0.18_{\text {stat }} \pm 0.55_{\text {syst }}$ & $186.12 \pm 4.91_{\text {stat }} \pm 11.09_{\text {syst }}$ & \\
\hline
\end{tabular}

Table 2: Fit results for the quadratic, pole and dispersive parametrizations of the form factors.

NA48/2 is the first experiment measuring the form factors using both $K^{+}$and $K^{-}$. The $K_{\mu 3}$ result is dominated by the statistical error, the $K_{e 3}$ by the systematic (background subtraction). The $K_{\mu 3}$ and $K_{e 3}$ are in agreement within each other and our combined results are competitive with the current world average. In order to avoid the problem of partially correlated systematic uncertainties 
in the averaging of the $K_{\mu 3}$ and $K_{e 3}$ results, the complete analysis has been repeated considering the two decay modes as a unique data set, containing two Dalitz plots simultaneously fitted with a common set of form factor parameters. The final results of the fit for quadratic, pole and dispersive parametrizations are again listed in Table 2 . All the measured parameters are in good agreement with the previous measurements done by other experiments.

\section{References}

[1] J.R. Batley et al. (NA48/2), Eur. Phys. J. C 52, (2007) 875.

[2] V. fanti et al. Nucl. Instrum. Meth. A 574, (2007) 433.

[3] L.M. Chounet, J.M. Gaillard, M. Gaillard, Phys.Rept. 4, (1972) 199.

[4] C. Patrignani et al. (Particle Data Group), Chin. Phys. C 40, (2016) 100001.

[5] P. Lichard, Phys. Rev. D 55, (1997) 5385.

[6] V. Bernard, M. Oertel, E. Passemar, J. Stern, Phys. Rev. D 80, (2009) 034034.

[7] C. Gatti, Eur. Phys. J. C 45, (2006) 417. 